\title{
Itis is Impact of Childhood Trauma Histories Versus Recent Trauma Symptoms on Affective Lability in Adult Bipolar Disorder
}

\author{
Amir Garakani, MD ${ }^{a, b, c}$; Frank D. Buono, PhDa; Kaitlyn Larkin, BA ${ }^{a}$; and Joseph F. Goldberg, MD ${ }^{c, *}$
}

E arly life adversity is a recognized predictor of poor outcome in mood disorders. Additionally, genetic factors may contribute to affective instability and emotional lability as a dimension across psychiatric conditions. ${ }^{1} \mathrm{~A}$ recent large study reported that affective instability and mood lability may be an indicator of a bipolar diathesis, as shown in the BRIDGE-MIX-II study. ${ }^{2}$ It remains unclear whether recency of trauma symptoms differentially impacts mood episodes or affective ability in adult bipolar patients. ${ }^{3-5}$ We therefore examined childhood trauma and current traumatic stress ratings in bipolar and unipolar inpatients, hypothesizing that higher levels of both childhood trauma and current trauma symptoms would be associated with more severe affective lability.

\footnotetext{
aDepartment of Psychiatry, Yale University School of Medicine, New Haven, Connecticut

${ }^{b}$ Department of Psychiatry, Greenwich Hospital, Greenwich, Connecticut 'Department of Psychiatry, Icahn School of Medicine at Mount Sinai, New York, New York

*Corresponding author: Joseph F. Goldberg, MD, 128 East Ave, Norwalk, CT 06851 (joseph.goldberg@mssm.edu).

J Clin Psychiatry 2021;82(5):21br13912
}

To cite: Garakani A, Buono FD, Larkin K, et al. Impact of childhood trauma histories versus recent trauma symptoms on affective lability in adult bipolar disorder. J Clin Psychiatry. 2021;82(5):21br13912.

To share: https://doi.org/10.4088/JCP.21br13912

(c) Copyright 2021 Physicians Postgraduate Press, Inc.

\section{Methods}

Participants $(\mathrm{N}=109)$ were consecutive, voluntary, English-speaking, non-intoxicated adult inpatients at a private psychiatric facility who provided written informed consent. Analyses were a post hoc exploratory investigation in this previously described cohort. ${ }^{6,7}$ Procedures complied with ethical standards on human experimentation and the 1975 Helsinki Declaration. The protocol was approved by the Western Institutional Review Board (Olympia, Washington). Diagnostic assessments included a modified semistructured interview version ${ }^{7}$ of the Mood Disorders Questionnaire, itself a bipolar disorder screening tool ${ }^{8}$; other ratings included the Quick Inventory for Depressive Symptomatology-Self Report (QIDS-SR), ${ }^{9}$ the 54 -item Affective Lability Scale (ALS),${ }^{10}$ the PTSD Checklist-Civilian (PCL-C), ${ }^{11}$ and the 28-item Childhood Trauma Questionnaire (CTQ). ${ }^{12}$

\section{Results}

Figure 1 reveals significantly and uniformly higher ALS subcomponent scores in bipolar than nonbipolar subjects.

Linear regression examined associations between ALS (the dependent variable) and PCL-C scores, while controlling for CTQ subscales (sexual abuse, emotional neglect, physical abuse, emotional abuse), bipolar/ unipolar diagnoses, QIDS-SR scores, and age at onset. The model was significant $\left(R^{2}=0.504, F_{9,38}=3.279, P=.007\right)$. Bipolar diagnoses $(P=.035)$ and PCL-C scores $(P=.047)$

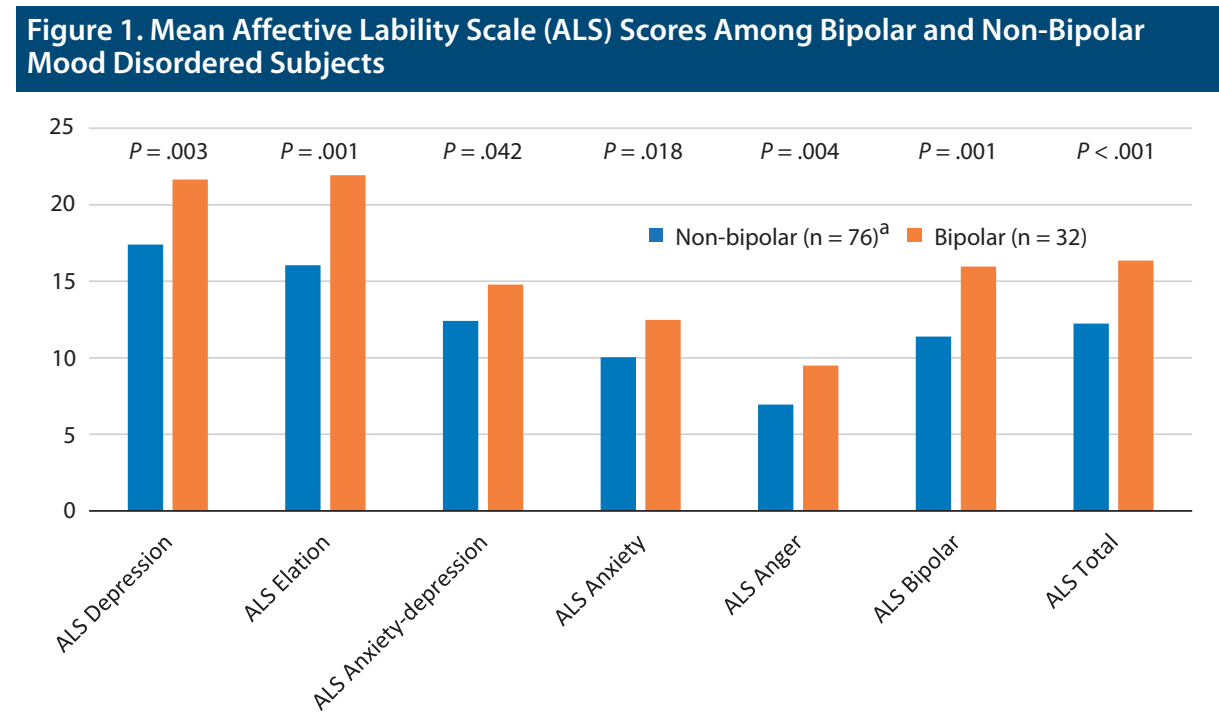

${ }^{a}$ One non-bipolar patient had incomplete ALS data. 
It ic illach thenct thic convwiol hto Figure 2. Regression Plot of PTSD Checklist-Civilian (PCL-C) Scores by Affective Lability Scale (ALS) Scores

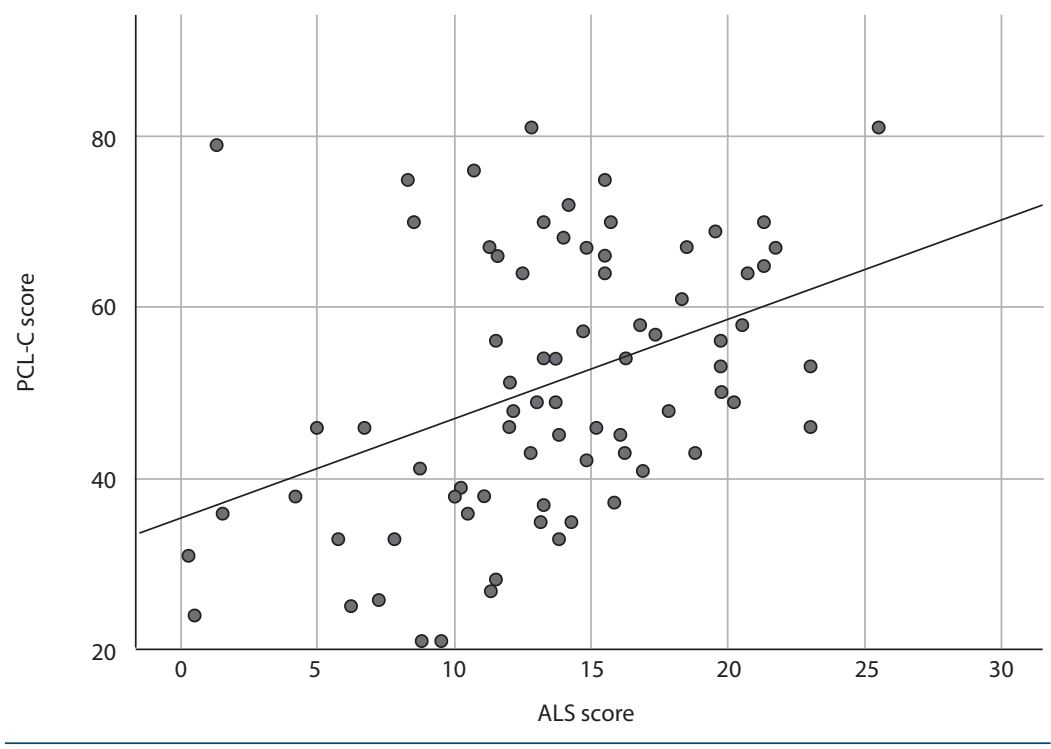

significantly predicted ALS total scores while controlling for age at onset $(P=.036)$. QIDS-SR total scores were significantly associated with ALS scores in univariate analyses but not when controlled for in the regression. No individual CTQ scores in the model were significantly associated with ALS scores. Current substance misuse did not significantly correlate with ALS scores or improve the explanatory power of the model. Figure 2 depicts the regression plot between PCL-C scores and ALS scores.

The present findings indicate a strong link between current distress about trauma symptoms and affective lability among inpatients with bipolar (but not unipolar) disorder, while controlling for current depressive symptoms or age at onset. Unexpectedly, self-reported histories of childhood trauma per se did not contribute significantly to ALS scores, despite prior literature linking childhood trauma with affective lability in adult bipolar disorder. ${ }^{1-3,13}$ These provisional findings require corroboration with larger sample sizes and longitudinal assessment, direct evaluation of adult trauma, and closer evaluation of the timing of adult versus early life trauma vis-à-vis current affective symptoms. While the PCL-C is used to screen for PTSD, formal DSM-5 PTSD diagnoses were not ascertained. The study also did not directly compare childhood vs adult trauma and instead focused on childhood trauma versus recent distress about trauma (using the PCL-C), as we did not include measures of adult trauma. Study subjects were self-selected for seeking treatment at a private psychiatric hospital, limiting generalizability. Most participants had comorbid substance use disorders. There is also the possibility that the presence of symptomatic bipolar or mood disorders would impact scores on the ALS and PCL-C and be a source of bias. Other limitations include potential recall biases and the impossibility to establish causality.

Future studies to discriminate the proximity and nature of childhood versus adult trauma, directly measuring both childhood and adult trauma, using validated scales and instruments, relative to $\mathrm{BD}$ mood episodes may help to shed further light on possible catalysts for affective lability and episodes within the kindling model. ${ }^{14}$

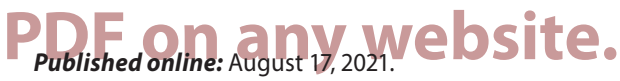

Potential conflicts of interest: Dr Goldberg has served as an advisor or consultant to BioXcel Therapeutics, Lundbeck, Otsuka, Sage, and Sunovion; served on the speaker's bureau for Allergan, Intracellular Therapies, Otsuka, and Sunovion; and received royalties from American Psychiatric Publishing, Inc, and Cambridge University Press. Drs Garakani and Buono and Ms Larkin have nothing to disclose.

Funding/support: This research received no specific grant from any funding agency, commercial or not-forprofit sectors.

\section{REFERENCES}

1. Gisbert L, Vilar L, Rovira P, et al. Genome-wide analysis of emotional lability in adult attention deficit hyperactivity disorder (ADHD). Eur Neuropsychopharmacol. 2019;29(6):795-802.

2. Verdolini N, Menculini G, Perugi G, et al; BRIDGE-IIMIX Study Group. Sultans of swing: a reappraisal of the intertwined association between affective lability and mood reactivity in a post hoc analysis of the Bridge-II-MIX study. J Clin Psychiatry. 2019:80(2):17m12082.

3. Marwaha S, Gordon-Smith K, Broome M, et al. Affective instability, childhood trauma and major affective disorders. J Affect Disord. 2016;190:764-771.

4. Aas M, Aminoff SR, Vik Lagerberg T, et al. Affective lability in patients with bipolar disorders is associated with high levels of childhood trauma. Psychiatry Res. 2014;218(1-2):252-255.

5. Aas M, Henry C, Bellivier F, et al. Affective lability mediates the association between childhood trauma and suicide attempts, mixed episodes and comorbid anxiety disorders in bipolar disorders. Psychol Med. 2017;47(5):902-912.

6. Garakani A, Buono FD, Larkin K, et al. Parsing the effects of comorbid adult ADHD and substance misuse on affective lability in bipolar disorder. J Affect Disord. 2020;266:338-340.

7. Goldberg JF, Garakani A, Ackerman SH. Clinicianrated versus self-rated screening for bipolar disorder among inpatients with mood symptoms and substance misuse. J Clin Psychiatry. 2012;73(12):1525-1530.

8. Hirschfeld RM, Williams JB, Spitzer RL, et al. Development and validation of a screening instrument for bipolar spectrum disorder: the Mood Disorder Questionnaire. Am J Psychiatry. 2000;157(11):1873-1875.

9. Rush AJ, Trivedi MH, Ibrahim HM, et al. The 16-Item Quick Inventory of Depressive Symptomatology (QIDS), Clinician Rating (QIDS-C), and Self-Report (QIDS-SR): a psychometric evaluation in patients with chronic major depression. Biol Psychiatry. 2003;54(5):573-583.

10. Koenigsberg HW, Harvey PD, Mitropoulou V, et al. Characterizing affective instability in borderline personality disorder. Am J Psychiatry. 2002;159(5):784-788.

11. Blanchard EB, Jones-Alexander J, Buckley TC, et al. Psychometric properties of the PTSD Checklist (PCL). Behav Res Ther. 1996;34(8):669-673.

12. Bernstein DP, Stein JA, Newcomb MD, et al. Development and validation of a brief screening version of the Childhood Trauma Questionnaire. Child Abuse Negl. 2003;27(2):169-190.

13. Marwaha S, Briley PM, Perry A, et al. Explaining why childhood abuse is a risk factor for poorer clinical course in bipolar disorder: a path analysis of 923 people with bipolar I disorder. Psychol Med. 2020;50(14):2346-2354.

14. Aldinger F, Schulze TG. Environmental factors, life events, and trauma in the course of bipolar disorder. Psychiatry Clin Neurosci. 2017;71(1):6-17. 